\title{
Multiagent Perspectives to Agile Scheduling
}

\author{
R. J. Rabelo ${ }^{1}$, L. M. Camarinha-Matos ${ }^{2}$, H. Afsarmanesh ${ }^{3}$ \\ ' Federal University of Santa Catarina, Department of Mechanical Engineering, \\ Florianópolis (SC) Brazil, rabelo@gsigma-grucon.ufsc.br. \\ ${ }^{2}$ New University of Lisbon, Faculty of Sciences and Technology, Monte da Caparica, \\ Portugal,cam@uninova.pt. \\ 3 University of Amsterdam, Department of Computer Science, Amsterdam, The \\ Netherlands,hamideh@wins.uva.nl.
}

\begin{abstract}
This paper focuses on the discussion of innovative and balanced perspectives of multiagent approaches to advanced agile scheduling systems in a system called MASSYVE. Several achieved results and current developments are described, taking as the basis the HOLOS framework. This framework is briefly described, emphasizing the use of negotiation approach to improve the scheduling flexibility, as well as the aspects of information integration, communication and coordination in a community of agents. For information integration and management in MASSYVE, three approaches are addressed, suggesting the utilization of the PEER distributed/federated database management system. Finally, some innovative aspects for agile scheduling are introduced, in specific to support it within the virtual enterprise environment.
\end{abstract}

\section{Keywords}

Agile scheduling, multiagent systems, information integration, virtual enterprise

\section{INTRODUCTION}

Global competitiveness has been imposing deep changes to all components of manufacturing systems, including the scheduling systems. From an emphasis on the scheduling optimality in the past, the focus has later on moved to scheduling flexibility, and now the emphasis is put on the support to scheduling agility. An agile scheduling system is able to both react dynamically in the presence of events not previously foreseen in the current schedule, and to consider the whole enterprise's production resources beyond their traditional shop floors' physical boundaries. The first property characterizes the dynamic scheduling and leads to the need of a precise definition for the borders and close integration of the production planning, the scheduling, and the execution supervision activities. The second property leads the enterprises to enlarge their business flexibility when supporting the virtual manufacturing and the virtual enterprise paradigms (Rabelo 96).

The original version of this chapter was revised: The copyright line was incorrect. This has been corrected. The Erratum to this chapter is available at DOI: 10.1007/978-0-387-35390-6_58 
Trends in agile scheduling can be analyzed under many perspectives. This paper aims to discuss the innovative perspectives for some multiagent approaches to advanced agile scheduling systems. It introduces some solutions and approaches to face one of the biggest general problems in the development of advanced scheduling systems, which is the fact that they require advanced information technology (IT) that is changing progressively. Furthermore, the integration of human-based decision-making and scheduling functionalities are also necessary. Therefore, the challenge in innovative scheduling is to propose balanced ways to extend the scheduling systems' life cycle, keeping them advanced to support the new emerging production paradigms and the new socio-organizational structures in such a technologically dynamic scenario (Smith 94).

The research described in this paper is being partially supported by the MASSYVE INCO-DC KIT Project, a cooperative initiative sponsored by the European Union, which involves Portugal (New University of Lisbon and the CSIN software-house), The Netherlands (University of Amsterdam), and Brazil (Federal University of Santa Catarina). MASSYVE (Multiagent Agile manufacturing Scheduling SYstems for Virtual Enterprises) (Massyve 97) aims to investigate the use of multiagent systems in agile scheduling, towards the operation in a virtual enterprise environment. These activities use the HOLOS framework (Rabelo 97) as a baseline for advanced scheduling. Some of the perspectives presented in this paper are already implemented in HOLOS, while some others are being analyzed and implemented in the scope of the MASSYVE project. The information integration approach to support multiagent systems in MASSYVE are based on the PEER information management framework (Afsarmanesh 94).

\section{HOLOS AGILE SCHEDULING SYSTEM}

Before discussing the new challenges raised by a virtual enterprise, the characteristics of the HOLOS system will be summarized. HOLOS was initially designed to support individual manufacturing enterprises. In the next chapters, extensions to this system towards a virtual enterprise will be discussed.

\subsection{The HOLOS Framework}

HOLOS is a framework specially developed to derive "instances" of agile scheduling systems. A HOLOS instance is composed of a set of distributed agents, configured for a particular enterprise, integrated with the local production resources, and which perform the scheduling and some level of execution supervision by means of the information and control interchange.

In this framework an instance of a scheduling system is derived from a reference model, the HOLOS Generic Architecture (HOLOS-GA) (Rabelo 95), that contains the general description of the agents' classes (i.e. the domain knowledge and the agents' attributes and functionalities). The architecture of HOLOS-GA is object-oriented, using the constructs introduced in Object Oriented Programming, and the power of the emergent approach of Agent Oriented Programming (Shohan 93).

The derivation of an instance of HOLOS-GA for an enterprise is a complex process. There are so many aspects to be considered and adapted, involving the areas of production planning, engineering, communications, information integration, etc., that it cannot be achieved by a simple copying of a derivation from one enterprise to another. 
The development of a derivation requires a supporting methodology. The HOLOS Methodology (Rabelo 96) corresponds to a sequence of steps and recommendations to be followed towards the implantation of an agile scheduling system in an enterprise. In order to cope with the mentioned complexity, the HOLOS framework includes the HOLOS System Generator (HOLOS-SG) (Rabelo 96). The HOLOS-SG is an interactive system that "automates" most of the steps of the methodology, but also considers the human expert's contribution, therefore leading to a semi-automatic derivation of instances of agile scheduling systems based on the HOLOS-GA. The system derivation philosophy has been considered as a balanced trend for the development of complex systems (Szelke94). In the case of HOLOS, it allows a system to be custom-tailored for a very particular enterprise and, at the same time, to be easily reconfigured and adapted whenever new production methods, algorithms, production resources, etc., are introduced or changed. Such modifications only require small alterations in the current version of the scheduling system and will not affect the control architecture of the whole system.

\subsection{The Multiagent Approach}

The Multiagent System (MAS) paradigm represents one of the most promising approaches to build complex and flexible advanced intelligent systems. The application of a MAS approach in agile scheduling is based on the idea that the scheduling agility can be extremely improved once it is based on the following key points: i) distributed and autonomous systems instead of the centralized and non-autonomous solutions; ii) negotiation-based decision making instead of the totally pre-planned processes; iii) use of different problem-solvers in the same environment instead of only one fixed problem solver; and iv) concurrent execution instead of the sequential processing (Tonshoff 95). In summary, a multiagent scheduling system is composed of a set of "processors" (nodes in a network of manufacturing resources), each one with its own particular capabilities (typically heterogeneous), that have to exchange and process information in order to contribute to finding a solution to the global scheduling problem.

In spite of the lack of a common definition in the literature, a processor is considered as an agent when it possesses at least the following three properties (Sichman 92): i) a certain degree of autonomy to reason about and to make decisions by itself; ii) the capability to interact with other agents; and iii) the knowledge to solve a part of the global problem independently. An agent can play several roles and behave in many different ways when it shows these elementary properties. One role it can play is to act cooperatively, that is the essential HOLOS agents' behavior, instead of acting destructively or selfishly. Cooperative scheduling ascends in significance in the complex manufacturing environments, while it is usually highly constrained. This means that a feasible and robust multiagent scheduling can only be generated and executed with the dynamic, flexible and intelligent relaxation of the constraints within the distributed agents, i.e. with real cooperation. The more efficient this cooperation process is, the more efficient the agile reaction of the entire production structure will be, and hence a better information quality is provided to support a rapid decisionmaking. However, when the manufacturing process is extended towards a VE environment, this cooperative assumption cannot be necessarily guaranteed. 


\subsection{Negotiation in Scheduling}

Several problems can arise during the schedule generation, after its generation, and during its execution, such as the temporal, capacity, or technologic conflicts. These problems come from the planning, scheduling or execution supervision activities. There are several methods that can be applied for the conflict resolution in a multiagent system. HOLOS uses the Contract-Net Protocol coordination mechanism to support the task assignment among the agents, and the Negotiation (Davis 83) method to overcome conflicts taking place during one of the three above mentioned scheduling phases.

Figure 1 illustrates how the negotiation approach is used in HOLOS. Notice that the main function of a scheduling system is to assign tasks (production orders) to production resources (robots, $\mathrm{CNC}$ machines, workers, etc.) during certain periods of time. The production resources are represented by agents. Thus, the procedure is to (a) announce a task (an "enterprise activity", which is modeled as an object) through the MAS network and then making the agents exchange information about it with other agents, and so that (b) one of them is selected to perform such task at the end of this process (Rabelo 94).

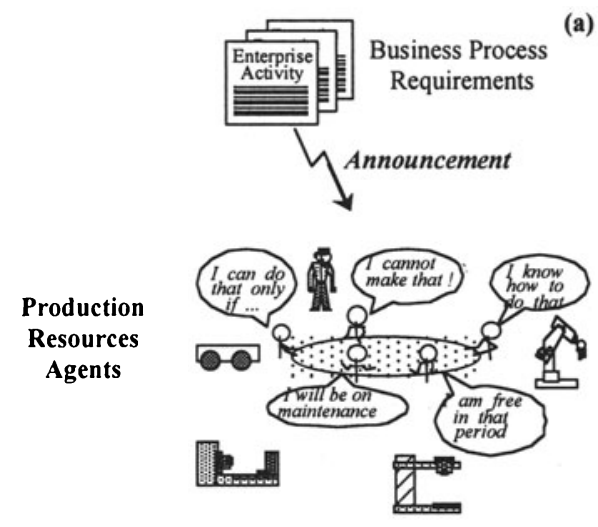

(a)

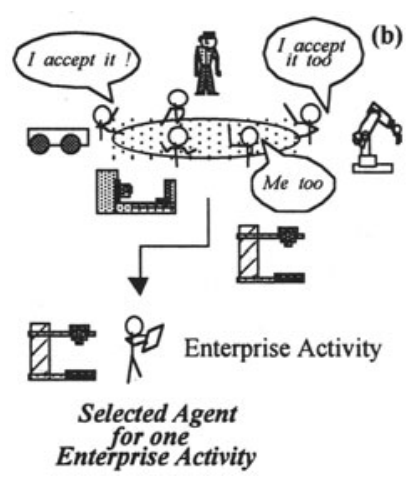

Figure 1 - Negotiation in scheduling.

The coordination of this entire network-based negotiation process comprises various phases and requires a specific high-level protocol. The HOLOS Negotiation Protocol corresponds to the protocol developed within this framework to support the information exchange associated with each of these phases. In terms of implementation, all the agents' knowledge base and the information exchanged among agents is modeled in Prolog and frames.

\subsection{Classes of HOLOS agents and their interactions}

A HOLOS agile scheduling system is a collection of classes of HOLOS agents configured for a particular shopfloor. Four classes of HOLOS agents described below are responsible to perform certain coordination tasks:

- Scheduling Supervisor (SS): is the class whose (unique) instance performs the global scheduling supervision. 
- Enterprise Activity Agent (EAA): is the class whose instances are associated to the production resources, i.e., resources are represented by EAA. These agents are the executors of tasks.

- Local Distribution Center ( $L D C$ ): is the class whose instances represent functional clusters of EAAs in order to avoid announcement broadcasting and hence to make the selection of an EAA faster. They are also responsible to select the most suitable agent for a certain task after the negotiation process.

- Consortium $(C)$ : is the class whose instances are dynamically created to supervise locally and in a decentralized way - the schedule of a logical arrangement of EAAs selected to execute an entire task (a "business process").

The EAAs are tile tasks executors, linked to "real entities", that "sell" the services that can be provided by the production resources they represent. The other agents are designed only for control and coordination purposes. The essential information control flow used in the negotiation-based scheduling is illustrated in the Figure 2. The CIM-IS represents a CIM Information System (see chapter 3).

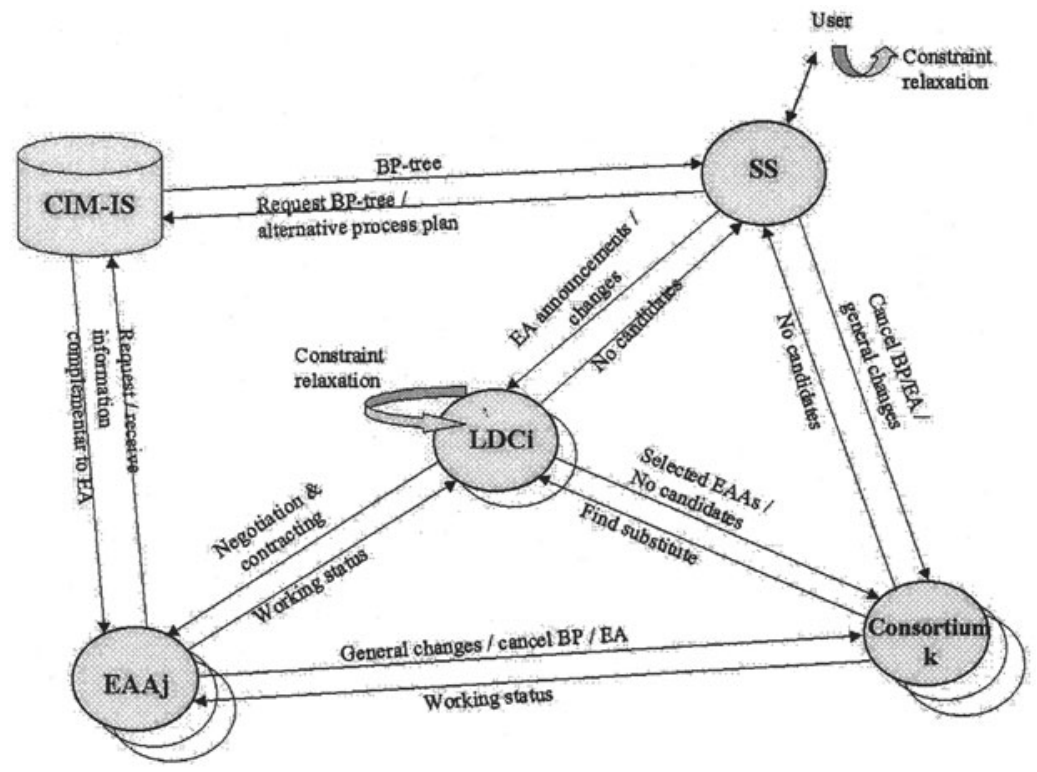

Figure 2 - Interactions between HOLOS agents.

Once launched in the computational environment, all the instances created by the HOLOS-SG become persistent, except the Consortium agents. A Consortium is alive and active as long as the task it supervises is not completed. Once the task is finished the respective Consortium dismantles itself. In HOLOS, there is no unique global and comprehensive schedule, but rather a collection of distributed and inter-related pieces of smaller schedules. The Consortium concept allows an enterprise to improve many aspects of manufacturing flexibility, including the internal routing, production, and 
shop floor organization. These three flexibility aspects provide a base to overcome some limitations of the Group Technology concept as well as to support the virtual manufacturing paradigm.

Since the topologic design of a multiagent control hierarchy has a high impact on the system performance, it is quite desirable to first evaluate it. The effects of a system's topology can be evaluated through measuring several types of costs, such as the execution (normally expressed in units of time wasted in the execution of a task), coordination, and vulnerability costs. In HOLOS the interaction between the agents is only vertical and agents cannot change the set of other agents that they can communicate with. Considering the classification schema proposed in (Malone 88), the HOLOS control hierarchy is functional with small processors, using one global manager (the agent SS), some functional managers (the agents LDC), and assuming that a shop floor is usually composed by production resources (the agents EAA) with small production capacities.

Inter-agents communication is a crucial aspect in MAS-based solutions. When it comes to implementation, this is a difficult aspect due to the strong heterogeneity in industrial environments. In the HOLOS scheduling environment, a HOLOS agent can establish a communication with four kinds of external entities: other HOLOS agents, the end-user, a CIM Information System and the production resources. Resorting to international or de facto standards in these communication processes, the information exchange becomes easier and faster. Another advantage that can be mentioned here is the increase of the system's life cycle, as there is no need to constant changes in the protocols and when new heterogeneous systems are added to the enterprise. Within HOLOS, the MAP/MMS (Mackiewicz 94) is used as the supporting high-level protocol for communication with the production resources, and the STEP/SDAI (Fowler 92) is used to communicate with the CIM Information System (CIM-IS). The HOLOS Negotiation protocol is used to support the high-level communication between agents. For example, "mm_LDContent (<machine_id>" is the MMS statement sent by a certain EAA to the production resource machine id to start the execution of a program stored in its memory. For the SDAI, the statement "is get_instance (process plan, $<$ pplan_id $>$ )" for instance, is used to access a certain process plan called <pplan_id $>$ in the CIM-IS. The statement "send_msg $\left(\langle\right.$ Consortium_id $\rangle,\left\langle S S \_i d\right\rangle,\langle\bar{O} C S$, $<$ task_id $>,\langle$ status $>$ ))" would be the answer of the agent $\langle$ Consortium_id $\rangle$ to the $<S S \overline{i d}\rangle$ regarding an earlier query about the status of a certain task. This message is semantically classified as "OCS" in the HOLOS Negotiation Protocol. Having different protocols that run simultaneously in the same environment requires an interoperation among protocols, that is handled in HOLOS.

The integration of the scheduling system with the production resources is however, a very demanding task, since many manufacturing devices are not open enough. In some cases it might even imply retrofitting some machines, e.g. due to the introduction of new sensors, new I/O functionalities, etc. This integration aims at making the EAA agents "talk" directly to the manufacturing resources (in MMS, in this case) in order to send commands and to receive their status, for instance in real-time. A balanced approach for the migration from existing components towards an integrated manufacturing infrastructure can be achieved via the "agentification" (a wrapping) of manufacturing resources (Camarinha-Matos 96). In other words, this agentification aims the transformation of manufacturing resources into agents that can cooperate in a multi-agent community (Figure 4). 


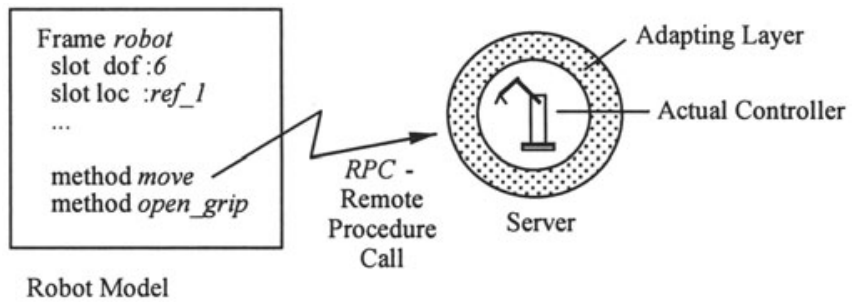

Figure 3 - The integration of existing controllers in an object-oriented framework.

This agentification process usually involves the following steps: i) building an adapting layer around the existing controllers in order to transform them into normalized servers; in the case of human resources, a human front end is necessary; and ii) building an agent manager, i.e. a high level "mirror" of the resource's functionalities.

As it can be noted, an EAA agent is, in fact, a "transparent" tandem aggregation of two processes (Figure 4): the server itself (as a wrapped resource controller) which executes the tasks, and the manager which manages the server's agenda and "sells" the server's capabilities to the agents community. The main advantage of this tandem architecture is its efficiency, due to the resulted parallelism that allows a speed up of the entire scheduling process. While the manager continues to negotiate the server's capabilities, the server keeps executing its contracted tasks.

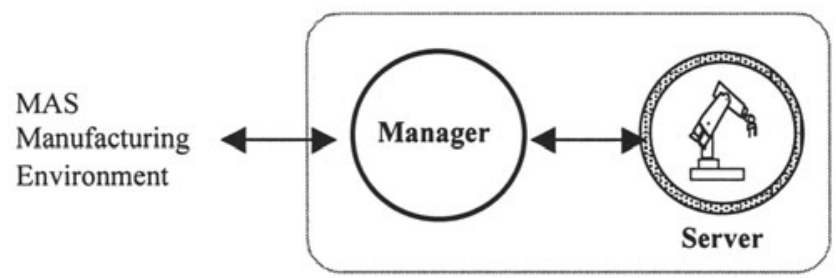

Figure 5 - Tandem-agent structure.

In terms of implementation, a HOLOS agent is a software module (computational process) modeled in frames, and having the following basic components:

- the manager (including a knowledge base, which contains the agent's functionalities), an agenda (which describes the list of scheduled tasks);

- a mailbox (which describes the tasks under negotiation);

- the communication protocol interfaces and;

- in the case of the EAA agents, the description of their capabilities.

Figure 5 shows the architecture of a HOLOS agent. The agents are implemented in Prolog extended with a frames layer, and the communication services are written in $\mathrm{C}$. A HOLOS scheduling system / agents run on Linux / PC machines.

Figure 6 illustrates a global view of a particular HOLOS multiagent scheduling scenario integrating the above concepts. The scheduling system is viewed as an application that receives / feeds data from / to a CIM Information System. Differently 
from classical systems, HOLOS is not a unique and comprehensive system, but rather a collection of distributed processes (agents). Each agent has its graphical user interface. In the EAA agents case, they are linked to the production resources they are associated to. For example, a certain EAA, identified as a "Robot_Scara", can establish a communication with the corresponding physical entity existing in the shopfloor.

\section{INFORMATION MANAGEMENT FOR MASSYVE SCHEDULING}

As it is mentioned in section 2.4, the architecture of the HOLOS system consists of the CIM-IS component for the integration and management of information among its agents. Through the CIM-IS, different systems in the enterprise can share and access all the information they need, in an integrated and transparent way, that is vital for the agile decision-making (see Figures 6 and 7). The CIM-IS can be regarded as the logical aggregation of the information structures (also called an "Integrated Schema" in a federated database architecture). In other words, the physical implementation of the CIM-IS can be achieved in several ways, e.g. with centralized or distributed/federated databases. In this section first the data and functional requirements of HOLOS scheduling is addressed. Then three approaches to the implementation of the MASSYVE information management is described. The PEER federated database system is also briefly described in section 3.3, since it is used in two of the implementation approaches considered for MASSYVE.

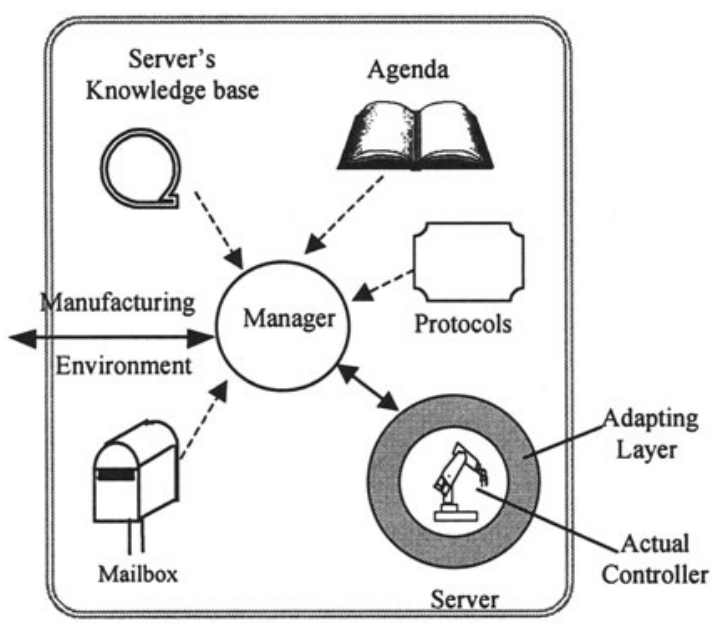

Figure 5-HOLOS agent architecture. 


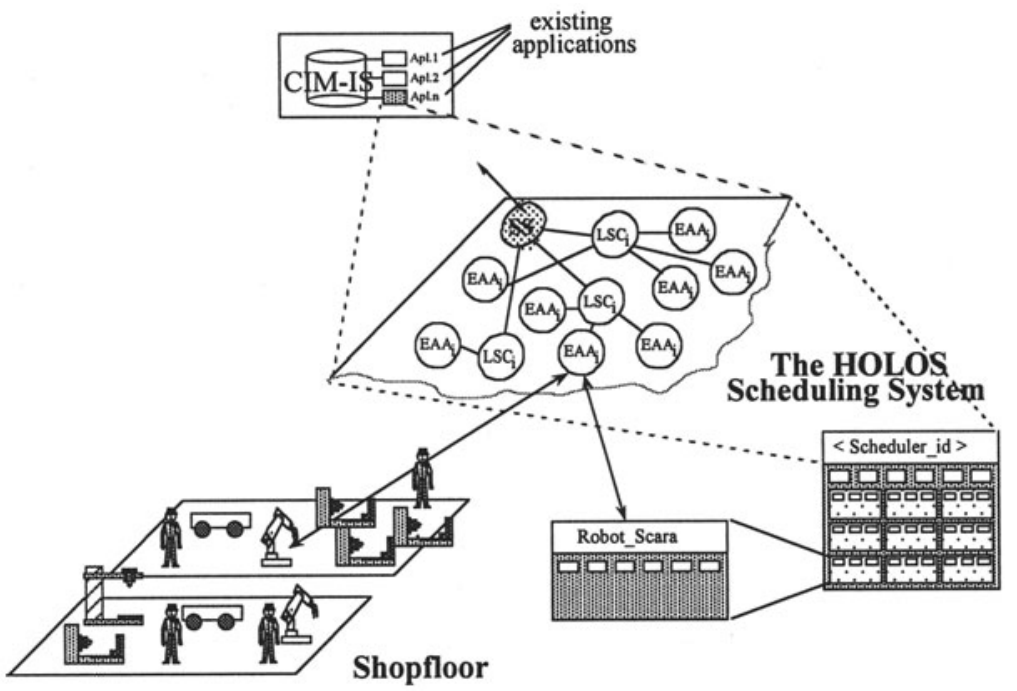

Figure 6 Example of a general MAS scenario.

\subsection{Variety of information sources for scheduling}

Agile scheduling requires access to a wide variety of information during both its generation and execution (see Figure 7). Some of this information is "indirectly" provided through the MRP activity of the Production Planning, while other information is collected directly from several other sources. At the same time, the information about the Production plan (or shop packet), the process plan, the product model, and the production resources models need to be gathered from several sources, in order to be used by the scheduling system.

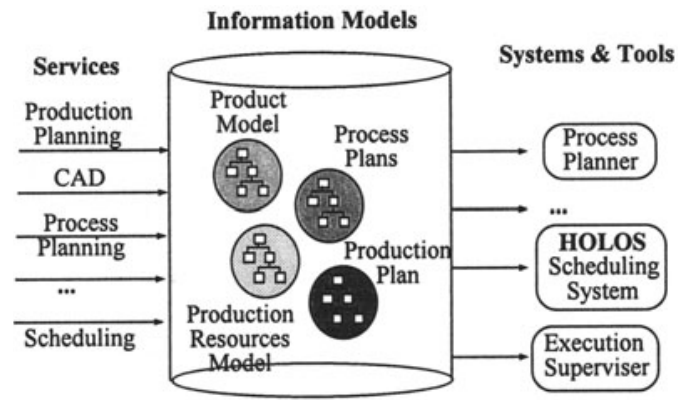

Figure 7 - The CIM-IS.

\subsection{Agent functionalities and their required data}

In the HOLOS architecture, every kind of agent plays a certain role, for which it requires to also access some data either from other agents, or from other sources of information. Some of the agents' functionalities are described and their required data is identified: 
- A scheduling starts when the SS agent consults the CIM-IS to check if there is a production plan with the list of tasks that needs to be scheduled. During the scheduling execution, the process plan models need to be accessed.

- The SS agent is the only agent that is visible outside HOLOS. Any other system, tool, or service, which needs to communicate with the scheduling system and access any scheduling related data, must contact the SS agent.

- Besides the SS agent, the only other kind of agent that has direct access to the CIM-IS is the EAA. The EAA agents access the CIM-IS during the scheduling generation, in order to get information about the tools, NC program, etc., necessary to perform their tasks.

- Once the scheduling system is derived, the production resources model is not needed anymore for the scheduling purposes. But the execution status of the production resources are needed to be accessed by the EAAs. Also the "scheduling models" (containing both the general information about the schedule of the tasks and the general status of the EAAs) must be made available in the CIM-IS, in order to be accessed by other activities / tools. The capability status is also needed to be stored in the CIM-IS, since they can change during the life time of the EAAs.

- Several other activities, for instance the planning, need to have up-to-date information about the schedules in order to modify the enterprise's production strategy and to guide the enterprise's logistics plan, that is fundamental in a global and competitive economy.

\subsection{PEER Federated database system}

The PEER federated database system, developed at the University of Amsterdam is an object-oriented information management system. It supports the sharing and exchange of information among cooperating autonomous and heterogeneous agents (or nodes) without the centralization and/or data-redundancy. In PEER, interdependencies between two nodes' information are established through the conceptual schemas defined on their information; thus there is no need to store the data redundantly in different nodes. Every node is represented with several schemas. A prototype of the PEER system is developed in the C language in UNIX environment.

\subsection{Different Approaches to information integration in MASSYVE}

The agile scheduling system, as described in HOLOS, requires an advanced database management system that supports all the requirements described in sections 3.1 and 3.2 above. Depending on the configuration of the environment, different approaches may prove to be more appropriate. In this section, three implementation architectures are presented and some of their characteristics are briefly discussed.

Centralized CIM-IS in HOLOS. In the existing implementation of the HOLOS system, a centralized implementation strategy is utilized, that sufficiently and efficiently supports the information access from different systems inside one enterprise. However, as with any centralized database, and depending on the size and complexity of the enterprise, there are certain disadvantages associated to this approach that can be better resolved for MASSYVE, if considering a distributed / federated architecture. A main problem is the vulnerability of the CIM-IS system, due to the centralization of all data in one node. A bigger problem will also rise in the case of a virtual enterprise, where a group of enterprises are involved. In such an environment, the centralization becomes a 
real bottleneck, both in the technical and organizational sense (see next section). Another problem is the inefficiency, which will rise in the case of a big enterprise that constitutes several production sites. In this case, the CIM-IS needs to manage the integration of large amount of updated data from several physically distributed sources, as well as the large number of information access requests from those systems (see the last section).

Federation of Virtual enterprises. A virtual enterprise is a group of enterprises that constitute physically and geographically distributed sources of information and/or systems. These enterprises need to cooperate and exchange information in order to jointly fulfil certain specific business opportunity. While intra-enterprise scheduling can be supported by the centralized approach described above, the centralized architecture is insufficient for the inter-enterprises scheduling. This environment is by nature distributed, and further consists of autonomous nodes. The PEER federated database architecture can properly support this complex domain (Afsarmanesh97). In this case, every enterprise will constitute a node in the federation, which maintains its local autonomy on the data and defines a set of export schemas through which the data is made available to other specific nodes. Also, every node will be able to import data from other nodes through their import schemas, and access their data according to the pre-defined access permissions. As a consequence of this general interaction facility, the approach allows the cooperation between the federated nodes in order to accomplish a common or global task, while the local autonomy and independence of every node is preserved and reinforced. Thus, there is no centralized repository of data or control and no need for data redundancy in the network.

Multi-layered Federated databases. Considering the future of manufacturing systems and enterprises, and in order to satisfy both the cases of virtual enterprises, and the case of big multi-site manufacturers, we propose a multi-layered federated database architecture. This architecture supports the sharing and exchange of information both within each multi-site enterprise and among different enterprises, uniformly. Clearly, the same architecture can also be applied within each enterprise (Afsarmanesh 97). If the components of an enterprise are distributed and run on different systems, the communication and exchange of information among different agents in HOLOS can benefit from the federated approach. Namely every agent, such as an EAA, can store and manage its generated information in its internal PEER database. Furthermore, different agents can share and exchange information via PEER to PEER access. In this architecture no centralization of information is required. The multi-layered federated database properly supports the evolution of the scheduling system through its life-time.

\section{EMERGING MULTIAGENT APPROACHES TO SCHEDULING}

The MASSYVE architecture is therefore based on the above described HOLOS and PEER systems. This chapter introduces further promising extensions to the HOLOS architecture in terms of emerging MAS approaches to negotiation-based scheduling (Figure 8). The extensions being evaluated in MASSYVE are divided according to three main perspectives: new organizational structures of multiagent systems, scheduling in a VE environment and contributions to workflow and project management. 


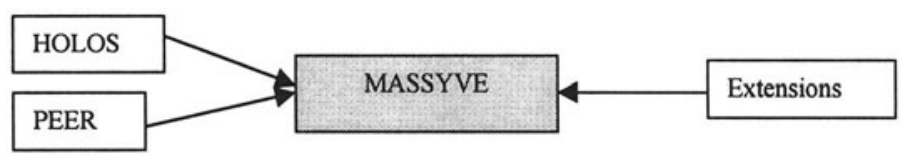

Figure 8 - Contributions to MASSYVE architecture.

\subsection{New MAS organization structures}

This topic refers to the introduction of a higher level of flexibility in the agents concerning their behavior in the hierarchical control structure. As mentioned in the section 2.4, the HOLOS structure is fixed in terms of types of interactions, agents' knowledge volatility and negotiation capabilities. However, several extensions can be added to the system, which can improve not only the quality of a schedule, but also the quality of the strategic, tactile and operational production plan. Examples of such extensions are:

a) Design of the organizational structure according to the needs of the application area. Other scheduling application areas can require other more efficient configurations of the multiagent system architecture and of the properties of their agents.

b) Support for dynamic assumption of roles by agents. The agents' pre-defined roles (i.e. their functionalities and levels of delegation) in a system can be dynamically changed or extended to deal with different tasks or situations, both in a virtual enterprise (VE) scenario and in an individual (local) enterprise.

c) Define criteria for selection of agents and their relation to the global optimization criteria. In HOLOS, the best bid from the (EAA) agents is selected by the LDC agents based on fixed criteria, using the classical contract-net philosophy. The levels of interaction / cooperation between these two classes of agents could be improved if the bidders could "iteratively" send to the LDCs their preliminary proposals (bids), asking if they are satisfactory or not, instead of sending final proposals at once. This can decrease the EAAs' local ignorance and can improve the quality of the LDCs' selection process. Another approach is to consider a stronger human intervention in this process, i.e., a less automatic process.

d) "What-if" supported negotiation. Like in MRP-II systems, the scheduling system can allow a schedule generation to be simulated (and hence evaluated) specially for capacity and logistic planning purposes. This means that EAAs would have to reason in two alternative "spaces": the real one or the simulated scenario. Eventually multiple scenarios might be kept during the what-if session.

e) Order splitting negotiation. In a classical scheduling scenario the splitting of orders is decided by the production planning activity. A different approach is to allow the EAAs (the scheduling activity) to offer splitting proposals based on their local agendas. This leads to an increase in the level of the agents' autonomy. This capability can be useful for a better resource management as a way to avoid bottlenecks, to decrease the risks caused by a machine failure or to improve flexibility in failure recovery, and to find EAAs when there is no one able to accomplish a full order.

f) Bargain in negotiation. Using a more sophisticated negotiation protocol, the EAAs can bargain with the LDCs about a certain order so that their agendas (i.e. the machine utilization) can be maximized. This also improves the agents' autonomy and the decentralized decision-making capability. 
g) Access to agents' internal status. Acting in a pro-active way, the high level supervisor agents could be permanently checking the status of their "slaves", even for those having reporting capabilities (because sometimes the agents can lie or become non operational). This feature can be applied both in a VE scenario and in an individual enterprise.

h) Organization for shared resources. EAAs (production resources) can work for several enterprises "simultaneously". In spite of a given production resource belonging to an enterprise, it could be with another one, like a resource rental.

i) Forecasting in Scheduling. Utilization of forecast information by the agents (mainly the EAAs) in order to improve their local schedules. In this sense, the agents can make bids also based on forecasting (seasonal products, pre-defined clients orders, weather conditions, etc.).

\subsection{Scheduling in a VE environment}

Under this section, some extensions to the scheduling system in order to cope with the VE environment are presented. Many of these facets can be also applied to the perspective presented in section 4.3, workflow and project management.

a) Cope with agents not totally cooperative. The goal of making two different enterprises cooperate using a MAS-based approach introduces a different situation regarding the willingness to cooperate. Although a general willingness to cooperate can be assumed when enterprises join their manufacturing efforts in a VE, variable levels of trust have to be considered. This means that the agents may be not cooperative, but rather selfish (they are not interested in a global optimization but only in themselves), or even destructive (competing enterprises, for instance, which send wrong information on purpose). The cooperation can be just one strategic behavior that a selfish enterprise, for example, can exhibit. Therefore, the agents may lie or even hide information.

b) Negotiation with incomplete or imprecise information. The classical negotiation protocols require, from the agents, a very well established sequence of complete information about a task in order to execute it. In several application domains, when an order is initially received it may show incomplete or imprecise data, being progressively complemented in a later phase (Camarinha-Matos 97). It is therefore necessary not only to adapt the agents and the protocols to allow reasoning with partial information, but also to prepare the production systems accordingly.

c) Global-contract-biased negotiation. MAS-based solutions have the local ignorance as an intrinsic problem. Local ignorance can lead an agent to misconsider useful information in its reasoning process which is related to a global negotiation contract (and not to individual orders). Therefore, more efficient knowledge propagation strategies have to be applied in order to provide all the involved agents with the necessary information.

d) Pre-defined or dynamic clusters of agents. The agents can dynamically change their relationship to some nodes instead of being associated to fixed groups of agents, including the agents of other enterprises.

e) Authority, accessibility to "internal" agents of a node, and organizational structure. In HOLOS, for instance, all interactions are vertical, i.e. agents of the same class (the same hierarchical level) cannot communicate with each other. However, in a wider scenario (like in a VE one) in which a global resource management is desired, horizontal interactions may be necessary. 
f) Volatility of agent skills. The agents' capabilities can change along their life cycle (new, change and/or loose of capabilities), which requires from the system as a whole a high degree of "self-adaptation".

g) Benefits of a VE schedule vs. individual schedules. A VE scenario requires a more cooperative relationship between its members than the one in traditional business relations between enterprises. So it makes sense to speak of a global VE schedule (global profits) instead of a collection of individual and independent schedules.

h) Competing scheduling. In a VE scenario, each enterprise normally has its own (local) schedule. However, depending on the task to be performed, each of the local schedules may "compete" with each other without considering a global gain. New strategies are necessary to handle this structure.

i) Distribution logistics scheduling in electronic commerce. Electronic Commerce is becoming more and more important. One of the bottlenecks for its implementation in some sectors is the distribution logistics. Specialized scheduling functionalities are required for this area.

j) Perturbation analysis (changes in behavior). In VE, the supply-chain has to be constantly monitored in order to guarantee realistic global schedules and to keep an updated logistic plan. In this sense, the agents / systems have to be sensitive to problems and to react accordingly.

k) Negotiation in VE using mobile-agents. Using this approach in the negotiation process means to "instruct" an agent about a "negotiation mission" to be accomplished in another enterprise. Thus, instead of making two enterprises to negotiate with each other being in their local sites, a mobile-agent can be moved to an enterprise to perform most of the automatic negotiation tasks locally. Once finished, it can be moved again to other enterprise of the VE to perform other negotiations, even taking the previous knowledge into account.

\subsection{Workflow and Project Management}

The general approach and extended facets described in previous sections can be generalized to other application areas. It seems particularly promising its application in the areas of workflow and project management systems.

In spite of the recent progresses in the workflow management systems, they are not very flexible in terms of dynamic changes of the workflow plans. Similarly, the area of project management support tools, specially when involving consortia formed by people from different organizations, can benefit from flexible negotiation-based scheduling and supervision. In particular, the introduction of dynamic scheduling, relaxation of constraints, tasks re-assignment, etc., are particularly promising here. Humans can be considered as a special kind of agents that need to be taken into account in a workflow execution or project management. When interacting with these agents, however, some of the basic assumptions of HOLOS, namely the assumption that agents are benign / totally cooperative, do not hold. Human agents may hide information or even lie for their own benefit. The generalization of the described approaches to these areas is another aspect being addressed by MASSYVE.

\section{CONCLUSIONS}

Multiagent systems and the negotiation-based task assignment represent an effective approach to the development of agile scheduling systems. A prototype system has 
shown the feasibility of the approach in the area of shop floor scheduling. The prototype includes also a framework for the derivation of particular architectures for specific manufacturing systems. In this paper, a number of possible extensions to the implemented architecture were identified in order to increase its agility and to support scheduling in a virtual enterprise environment.

One of the major improvements being analyzed is the integration of the multiagent architecture with a federated database management approach. This new research direction seems particularly suited to the requirements of a VE. Another challenge to previous approaches is the handling of agents that are not necessarily totally cooperative, as is the case in a virtual enterprise. Another key issue is the scheduling in the context of imprecise and incomplete task information, as a result of the management of incomplete and imprecise orders in a supply chain.

These aspects represent some of the issues being researched in the framework of the MASSYVE project.

\section{ACKNOWLEDGEMENTS}

This work is funded in part by the European Commission, project INCO-DC KIT MASSYVE number 962219.

\section{REFERENCES}

Afsarmanesh, H.; Wiedijk, M.; Hertzberger, L. (1994) Flexible and Dynamic Integration of Multiple Information Bases, Proceedings DEXA'94 $-5^{\text {th }}$ IEEE International Conference on Databases and Expert Systems Applications, Springer-Verlag, pp. 277-288.

Afsarmanesh, H; Garita, C; Hertzberger, L.O.; Santos, V. (1997) Management of Distributed Information in Virtual Enterprises: The PRODNET Approach - in proceedings of the 4th International Conference on Concurrent Enterprising (ICE'97).

Camarinha-Matos, L. M.; Rabelo, R.J.; Osório, L. (1996) Balanced Automation, in Management and Control of Manufacturing Systems, ed. S. Tzafestas, Springer-Verlag, pp. 376-413.

Camarinha-Matos, L. M.; C. Lima; Osório, L. (1997) The Prodnet platform for production planning and management in virtual enterprises, Proceedings ICE'97 $-4^{\text {th }}$ International Conference on Concurrent Enterprising, pp. 385-406, Nottingham UK.

Davis, R.; Smith, R. - Negotiation as a Metaphor for Distributed Problem Solving, Artificial Intelligence, N 20, pp. 63-109, 1983.

Fowler, J. (1992) Proposal for the STEP Data Access Interface Specification, STEP Implementation Specifications Committee, NIST.

Malone, T.; Smith, S. (1988) Modelling the Performance of Organisational Structures, International Journal on Operations Research, Vol 36 N 3, pp. 421-437.

Mackiewicz, R. (1994) An Overview on the Manufacturing Message Specification, in http://litwww.epfl.ch/ mms.

Massyve (1997) http://www.uninova.pt/ massyve.

Rabelo, R.J.; Camarinha-Matos, L. M. (1994) Negotiation in Multiagent Based Dynamic Scheduling, Journal on Robotics and Computer Integrated Manufacturing, Vol $11 \mathrm{~N} \mathrm{4,pp.}$ 303-310, Pergamon.

Rabelo, R.J.; Camarinha-Matos, L. M. (1995) A Holistic Control Architecture Infrastructure for Dynamic Scheduling, in Artificial Intelligence in Reactive Scheduling, Eds. Roger Kerr e Elizabeth Szelke, pp.78-94, Chapman \& Hall.

Rabelo, R.J.; Camarinha-Matos, L. M. (1996) Deriving Particular Agile Scheduling Systems using the HOLOS Methodology, International Journal in Informatics and Control, Vol $5 \mathrm{~N} 2$, pp. 89-106, Romania, June. 
Rabelo, R.J.; Camarinha-Matos, L. M. (1996) HOLOS : a methodology for deriving scheduling systems, em Balanced Automation Systems - Architectures and Design Methods, Eds. Luis M. Camarinha-Matos and Hamideh Afsarmanesh, Chapman \& Hall, pp. 181-194.

Rabelo, R.J. (1997) A Framework for the Development of Manufacturing Agile Scheduling Systems - A Multiagent Approach, Ph.D. Thesis, New University of Lisbon, Portugal.

Sichman, J.; Demazeau, Y. (1992) When can knowledge-based systems be called agents ?, Proceedings IX Brazilian Symposium on Artificial Intelligence.

Smith, S.; Lassila, O. (1994) Configurable Systems for Reactive Production Management, IFIP Transactions (B-15) on Knowledge-Based Reactive Scheduling, Eds. E. Szelke and R. Kerr, North-Holland, pp. 93-106.

Shoham, Y. (1993) Agent-Oriented Programming, Artificial Intelligence, N 60, pp.51-92, Elsevier.

Tonshoff, H.; Aurich, J.; Winkler, M. (1995) On The Way to Autonomous and Cooperative Manufacturing Systems, Proceedings $1^{\text {st }}$. World Congress on Intelligent Manufacturing Processes \& Systems, pp. 420-435, Puerto Rico.

\section{BIOGRAPHY}

Ricardo J. Rabelo received his B.Sc. on Computer Science in 1984 at the Federal University of Santa Catarina (Brazil), worked as a consultant for several Brazilian companies and finished his Ph.D. Thesis at the New University of Lisbon (Portugal) on Robotics and CIM, in 1997. His main interest areas are: agile scheduling, virtual enterprise, multiagent systems and information integration.

Luis M. Camarinha-Matos is associate professor at the New University of Lisbon where he coordinates the Robotics and CIM group. He has been involved, both as researcher and has technical coordinator, on several international research projects in the areas of virtual enterprises, multiagent systems, intelligent manufacturing systems and machine learning. He has served in the Program Committee of many conferences and was one of the founders of the BASYS conference series. He is the coordinator of MASSYVE.

Hamideh Afsarmanesh is an assistant professor at the University of Amsterdam in the Netherlands. She has been involved and has directed the research in several European (ESPRIT, and DUTCH-HPCN) and American funded projects. At the WINS faculty, she coordinates the research in the area of Cooperative and Federated Databases, and interoperable information management systems. She has served as the Program Chairperson in International Conferences and Workshops in the area of information management and expert systems. 